\title{
Forming of film surface of very viscous liquid flowing with gas in pipes
}

\author{
Krystian Czernek ${ }^{1, *}$ and Stanisław Witczak ${ }^{2}$ \\ ${ }^{1}$ Opole University of Technology, Faculty of Mechanical Engineering, Department of Process Engineering, 5 Mikołajczyka Street, \\ 45-271 Opole, Poland \\ ${ }^{2}$ Opole University of Technology, Faculty of Mechanical Engineering, Department of Process Engineering, 5 Mikołajczyka Street, \\ 45-271 Opole, Poland
}

\begin{abstract}
The study presents the possible use of optoelectronic system for the measurement of the values, which are specific for hydrodynamics of two-phase gas liquid flow in vertical pipes, where a very-highviscosity liquid forms a falling film in a pipe. The experimental method was provided, and the findings were presented and analysed for selected values, which characterize the two-phase flow. Attempt was also made to evaluate the effects of flow parameters and properties of the liquid on the gas-liquid interface value, which is decisive for the conditions of heat exchange and mass transfer in falling film equipment. The nature and form of created waves at various velocities were also described.
\end{abstract}

\section{Introduction}

Two-phase gas- very viscous liquid flow is quite common in technology applied in the up-to-date industry, both with regard to transport processes as well as thermal processing of liquid substances. The concurrent involvement of both gas and liquid phases with a very viscous in such systems results in a number of difficulties in ensuring adequate operating conditions for various types of apparatuses (heat exchangers, evaporators, reactors) in which the processes of exchange of momentum, heat and mass are realized. The basic reasons for such circumstances are associated with large stochastic characteristics of two-phase gas-liquid flow. Additionally, depending on the streams of the gas phase and liquid phases and the volume fractions of the liquid and solid phase various flow structures and forms of flow are encountered there. The process conditions corresponding to these structures constitute the fundamental obstacle in the process of ensuring maintenance adequate flow regimes and, hence, preservation of process parameters along the course of the flow.

In these circumstances important role is attributed to such solutions that ensure adequate operation of process apparatus within a maximum time frame. Concurrently, the solutions applied have to eliminate the disadvantageous phenomena associated with the course of the processes (instability of flow, origin of residue on the surfaces of the heating pipes in exchangers and evaporators, bubbly structures on liquid surface, instability of the interfacial surface, etc.). One of the ways in which solutions are sought is by indication of such solutions that enable the processes being realized in strictly defined flow regime, and, consequently, by indication of the possibility of conducting the processes of heat exchange within an most effective range of flow regimes.

The focus in this paper is on the issues associated with the possibilities of applying annular form of two-phase gas-liquid flow as one of the most effective ways in performing heat exchange processes, in particular with regard to heat and mass with a concurrent possibility of using substances whose physical properties are variable in the course of these processes. Phenomenological assessment of this type of flow is related to annular flow of gas and viscous liquid, whereas the latter term refers to such liquid in which the dynamic viscosity coefficient (at the temperature of $20^{\circ} \mathrm{C}$ ) exceeds the corresponding value for water.

The thin layer of liquid, also known as film, may be formed in various manners. For instance, in terms of the distribution of the liquid over the heating surface of evaporative apparatus, basic three manners are distinguished by Czernek [4]: static, mechanical and hydraulic.

In the consideration of the extensive range and various application of two-phase gas-liquid annular flow and, consequently, extensive application of various forms of it in apparatuses that apply patterns encountered in this type of flow, this paper focuses on the analysis of applicability of annular gas-liquid flow with regard to the most comprehensive and verifiable range of liquid viscosities possible to realize in original studies.

In the literature in this subject Czernek et al. [3, 5], Czernek [2, 4], Xu et al. [15], Oriol et al. [10], Jassim et al. [9] the items devoted to two-phase gas-very viscous liquid flow are relatively scarce despite the wide range of existing applications of such systems. The results

\footnotetext{
*Corresponding author: k.czernek@po.opole.pl
} 
presented up-to-date focus on examinations and descriptions of the phenomena occurring during flow of model mixtures, mostly involving air and aqueous solutions of sugar and polymers or oily liquids with relatively low viscosity. These papers involve identification and descriptions of flow structures, flow regime maps, pressure drops and determination of volume fractions of the particular components of the mixture, as well as thickness of liquid films and characteristics of their wavy motion. These issues are typical for the description of hydrodynamics of the twophase gas-liquid streams. A fair share in this research has been conducted by Department of Process Engineering, Opole University of Technology, where the work mainly focuses on two-phase gas-liquid flow of liquids with the viscosity of over $100 \mathrm{mPa} \cdot \mathrm{s}$, presented in papers Czernek et al.[3], Troniewski et al. [12], Troniewski et al. [13], Witczak et al. [14].

By definition, such research is conducted on systems containing two media, i.e. for air-oil mixture flow, which in most cases comes down to assessment of hydrodynamics of such flow with emphasis of the various aspects of it. However, due to the characteristics of the liquid phase (i.e. for a phase with a high viscosity), in such flow we have to do with different characteristics of the flow when compared with twophase single-component gas-liquid. Primarily, this is manifested by the occurrence of non-homogeneity of structures during flow of two-phase two-component mixture, which determine different volume fractions of these phases. Consequently, this leads to various hydrodynamic conditions along the flow route.

\section{Experiments}

The diagram of the experimental set-up applied for testing annular downward flow of gas-very viscous liquid is presented in Fig. 1. The media applied in this research involve air and machine oil with various viscosity coefficients. Flow channels 12 and 13 (with the diameter of $22 \mathrm{~mm}$ ) were fed with compressed air from a central pressure installation 4 . The airstream passed through a reducing valve 5 , and was regulated by means of a battery of rotameters 11 . Constant gas pressure was maintained through a reducing valve, which secured correct measurement of its stream. The air stream was subsequently directed into the feeding system in the front section of each of the channels that formed a system with a central nozzle. Liquid was fed from tank 2, which could hold around 80 litres of oil in total. The tank was equipped with a agitator and heaters (with the heating power of $8 \mathrm{~kW}$ ) with a thermostat that ensured maintenance of a constant temperature. Oil was pumped into the flow channel by means of a gear pump driven by a DC motor, which enabled the control system to adapt rotational speed of the pump continuously to ensure an adequate feed of oil stream. From the pump the oil was directed to a battery of rotameters 10 , which were used to assess the stream of this component of the mixture. Subsequently, the oil was pumped into the feed tank (the one containing gas phase) where the two-phase mixture was formed. The gas and liquid streams were selected in such ratios that ensured that annular flow of two-phase gas-liquid mixture was obtained at its output.

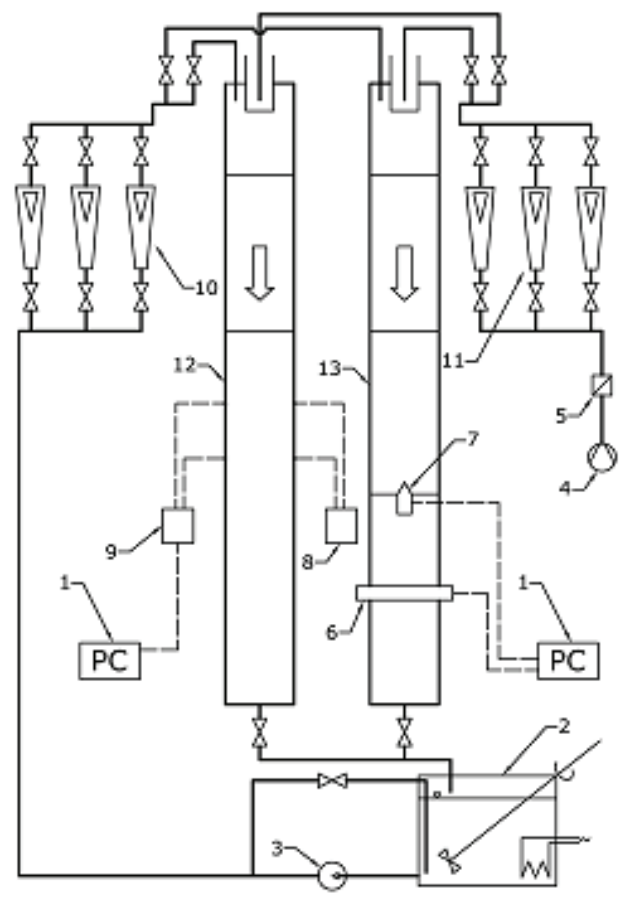

Fig. 1. Diagram of test stand: 1 - PC, 2 - oil tank with agitator and heater, 3 - oil pump, 4 - air compressor, 5 - reducing valve, 6 - laser knife, 7 - camera, 8 - laser illumination, 9 light detector, 10 - oil rotameter assembly, 11 - air rotameter assembly, 12 - measurement channel with laser illumination, 13 - measurement channel with laser knife.

In the same manner, other flow structures could be formed, which served for the purposes of comparison and search for the boundary between one structure and another. The measurement channel $(12,13)$ consisted of two parts - a non-transparent section and a transparent one. The non-transparent section (in the initial section of the pipe) ensured that stability of flow was obtained in the part of the channel, which served for the measurements. The latter was equipped with control and measurement instrumentation for optoelectronic evaluation of flow parameters $6 \div 9$. By means of these optoelectronic sensor linked to as PC (with a card for data storage and processing), it was possible to measure the local thickness of the liquid films and characteristics of their waves. A linear measurement system was applied for these purposes (Fig. 2) consisting of four single-axis measurement probes distributed in various cross-sections of the channel.

These probes apply the principle of light spectrum dissipation when crossing an oil film. In turn, the optoelectronic assembly 6,7 in this case formed a complementary part of the measurement system and applied the method of the so called light knife (Fig. 3), which enables the assessment of parameters that are specific for such flow in the cross-section of the channel. The mixture leaving the non-transparent section of the pipe was directed into the transparent section of the channel made of plexiglas. The observations regarding flow patterns were performed in this section and the 
structures are recorded by means of a digital video camera and a photo camera. Subsequently, the mixture passed on to the tank/separator 2 , in which two phases are separated.

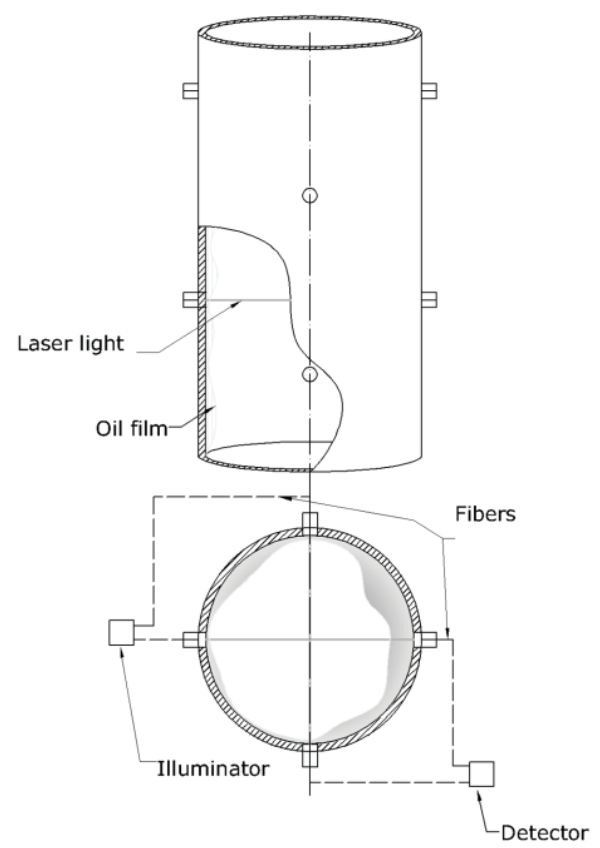

Fig. 2. Optoelectronic single-axis probe.

The applied measurement and control systems enable on to conduct research within a wide range of parameters of the flowing films. In the design phase of the research an attempt was made to select the range of the streams in a manner that ensure the occurrence of both laminar and turbulent flow in each of the measurement channels, for laminar liquid flow. Flow parameters were adapted throughout the research for air in the range $(1.8-384)$. $10^{-5} \mathrm{~m}^{3} / \mathrm{s}$ and for oil $(2.92-965) \cdot 10^{-7} \mathrm{~m}^{3} / \mathrm{s}$. The temperature of the mixture varied, accordingly, which was later accounted for during calculations of specific parameters.

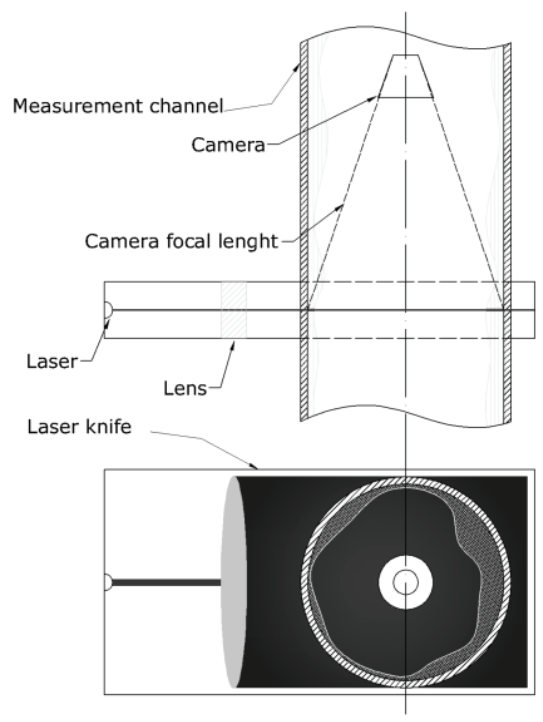

Fig. 3. Diagram illustrating the operating principle of laser knife.
The testing of the two-phase mixture flow were conducted in series for a dozen or so various air streams, which for each case was accompanied by several dozen measurements regarding oil streams for the preestablished flow parameters. During measurements conducted in this way simultaneous observations of the forming flow structures were made.

The circulating media applied in these hydrodynamic researches were air and oil. The rationale for this selection was associated with ensuring the possibility of gaining the highest possible value of dynamic viscosity coefficient. In order to obtain a wide range of oil viscosity, this research was performed for the range of temperatures $(10-40)^{\circ} \mathrm{C}$, for which the value of the dynamic viscosity coefficient varies in the range 90$3500 \mathrm{mPa} \cdot \mathrm{s}$. Low temperatures were obtained as a result of oil cooling until the liquidity state (around $10^{\circ} \mathrm{C}$ ). The gas phase applied in the testing was air, which was extracted from an external compressed air installation. An adequate measurement of its pressure and temperature enable one to determine its physical properties indispensable for conducting further calculations.

Rotameters were applied to adjust air stream. Prior to testing they were calibrated to ensure that it would be possible to read the values of the air stream directly from rotameters. In turn, to determine the characteristics of oil streams it was necessary to calibrate rotameters to match the specific liquid viscosities depending on their temperatures (adiabatic flow), as this value has a considerable effect on the efficiency of rotameters.

A digital video camera or a photo camera to provide a more comprehensible documentation performed the registration of the structures.

\section{Results and discussion}

The results of tests were applied for classification of flow patterns and verification of the flow regime map.

A special characteristic of two-phase gas-very viscous liquid flow is associated with the fact that for the identified patterns of annular flow or patterns that are similar to annular flow in terms of spatial distribution of the liquid and gas phase (AL, AW, AH regimes, see Fig. 4), the observed thickness of liquid film is definitely smaller than the diameter of the gas root. The remaining regimes noted for this case essentially correspond qualitatively to forms described in the literature for liquids with much smaller viscosity of the liquid phase. This was indicated by Andritsos et al. [1], whose research was performed within the range of liquid viscosity variability up to $80 \mathrm{mPa} \cdot \mathrm{s}$. In particular, this pertains to the aspect of the smoothening effect of rising liquid viscosity on the geometric configuration of the resulting patterns.

These remarks are further confirmed by author's original experimental research Troniewski et al. [12], Czernek et al. [5] conducted within the range of liquid viscosities up to $3500 \mathrm{mPa} \cdot \mathrm{s}$. An example of registered flow structure is presented in Fig. 4. 
Such a classification can be fully recommended as universal for the entire range of the occurrence of various flow patterns in a downward gas-very viscous liquid flow. In contrast to classification presented by Da Silva et al. [6], Geraci et al. [7], Hernandez et al. [8], Szalanski et al. [11] the novelty here involves the observation of a stalactite flow structure, in which the gas phase occurs in the liquid phase in the form of large bubbles with toroidal shape and a length that is comparable to the diameter of the channel and some of the liquid in the form of a thin stream is additionally transferred in the vicinity of the gas torus.

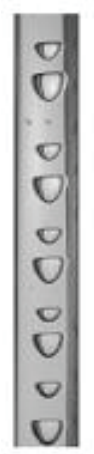

B

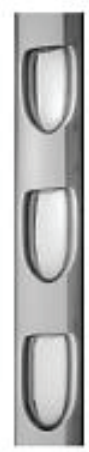

P

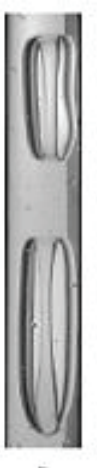

S

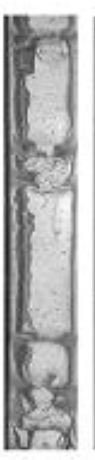

$\mathbf{F}$

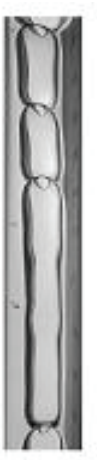

AR

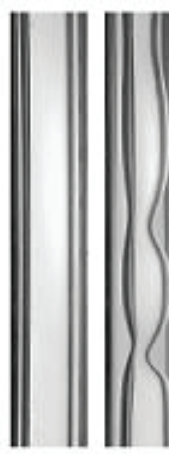

AL AW

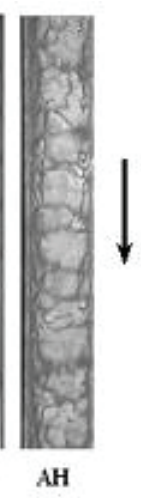

Fig. 4. Two phase gas-very viscous liquid downward flow structures: B - bubbly flow; P - plug flow; $\mathrm{S}$ - stalactite flow; $\mathrm{F}$ - foam flow; $\mathrm{AR}$ - annular core flow; $\mathrm{AL}$ - annular low wave flow; AW - annual wave flow; AH - annular hydraulic flow.

With regard to the structures of annular flow (AL-AH), for which the largest divergence was noted from the regimes occurring during flow of a liquid with varied viscosity, an independent identification of them was undertaken in order to specify the forms and range of their occurrence, as presented in Fig. 5

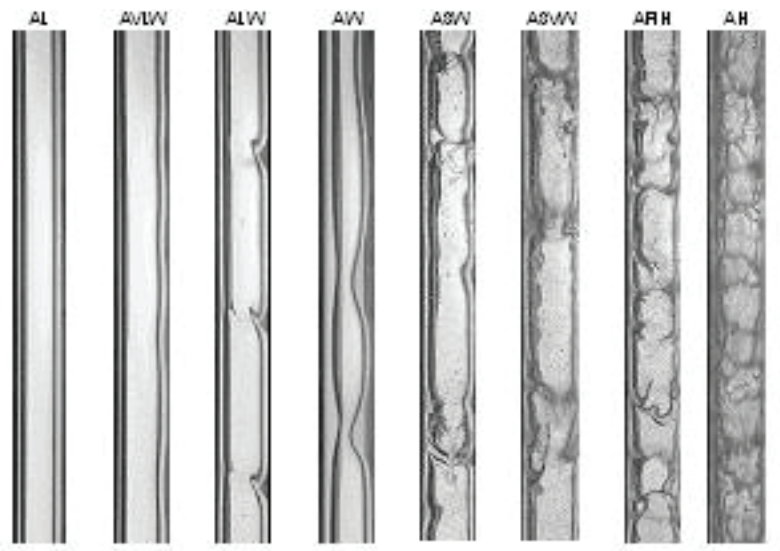

Fig. 5. Classification of two-phase annular gas-very viscous liquid downward flow patterns: AL-smooth film, AVLW-very low wavy annular flow, ALW-annular low wavy film, AW- wavy film, ASW- strongly wavy film, AVSW-very strongly wavy film, APIH-flow passing into hydraulic, AH-hydraulic film flow - for the range: $(90 \div 3500) \mathrm{mPa} \cdot \mathrm{s}$.

The rationale for the selection of this classification is associated with the fact that the structures resulting from it in each case are characterized by possibility of unequivocal assessment of some special characteristics of the flowing liquid film. It is possible to distinguish three basic ranges of such flow structures. The first one (AL-AVLW-ALW) is specific for smooth or slightly wavy interfacial structure surface, second one (AWASW) is always associated with the occurrence of waves in this surface and the third one - specific for hydraulic forms (ASVW-APIH-AH), with highly dispersed configuration of wave crests and occurrence of interference associated with formation of capillary waves.

In addition, one can note that the first group of flow structures (smooth film and low waves) occurs for small liquid velocities and for large range of gas velocities (in particular for a liquid with high viscosity). In turn, wavy structures are encountered for medium range of the values of these velocities, while hydraulic ones (irregular) forms occur when the values of phase streams, an in particular, ones of the gas, are in the highest range. In the latter case these phenomena are accompanied by a considerable effect of the condition of liquid viscosity - the lower the value of it, the more limited range of reciprocal overlapping of the waves (and the smaller formation of capillary waves).

The ranges of the occurrence of particular annular flow structures registered throughout this research have been identified on the basis of flow maps, whose examples are presented in Figs. 6-7 with regard to a channel $22 \mathrm{~mm}$ in diameter.

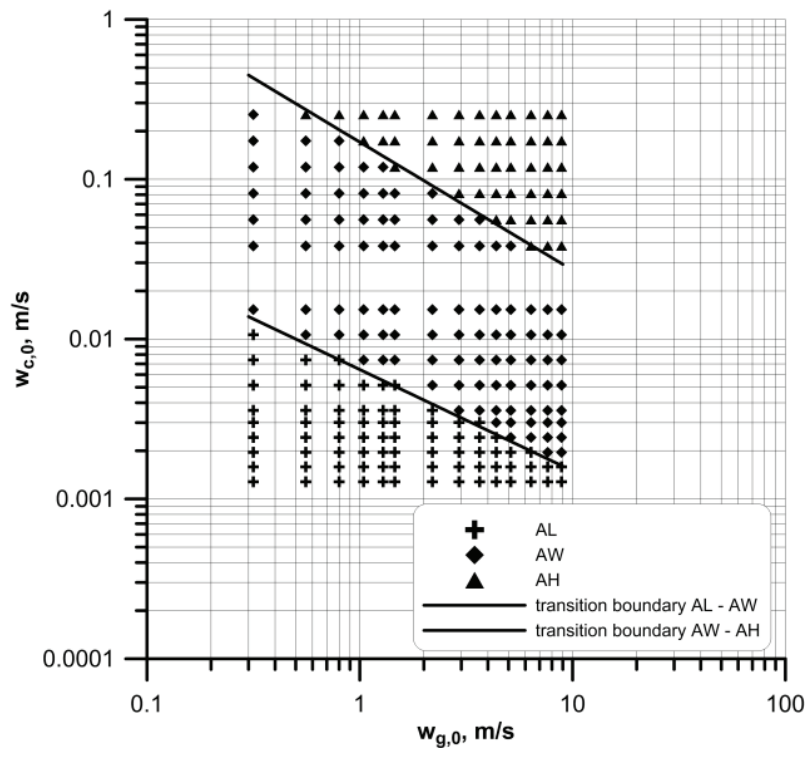

Fig. 6. Ranges of two-phase gas-high viscosity liquid downward flow in a pipe with the diameter of $22 \mathrm{~mm}$, and oil viscosity of $90 \mathrm{mPa} \cdot \mathrm{s}$.

The analysis of the occurrence of a given flow regime involved, subsequently, determination of the effect of liquid viscosity and phase streams (by reference to the superficial velocities) on the range of the specific structures formed. These maps also contain boundaries of the transfer between areas that are specific for smooth films and wavy structures (AL-AW transition boundary) and the ones between the wavy structures and 
completely hydraulic flow (AW-AH transition boundary).

The foundation for the assumption of the first boundary transition (AL-AW) was the appearance of waves on its surface with a geometry that could be described in terms of a specific wavelength and amplitude.

The passing of the other boundary $(\mathrm{AW}-\mathrm{AH})$ was taken for the case of occurrence of irregular waves, whose nature was difficult for unequivocal assessment.

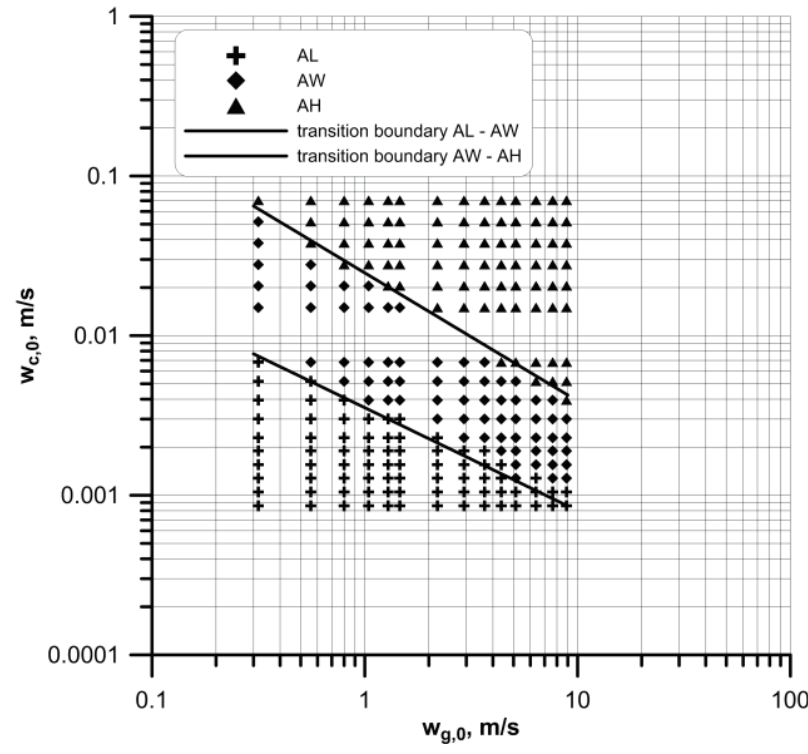

Fig. 7. Ranges of two-phase gas-high viscosity liquid downward flow in a pipe with the diameter of $22 \mathrm{~mm}$, and oil viscosity of $1520 \mathrm{mPa} \cdot \mathrm{s}$.

Qualitative and quantitative analysis indicates that it is possible to determine the ranges of occurrence of various structures of annular flow of very viscous liquid. This has led to the development of a generalized map of twophase gas-very viscous liquid flow, which is presented in Fig. 8 together with quantitative description of the transition boundaries.

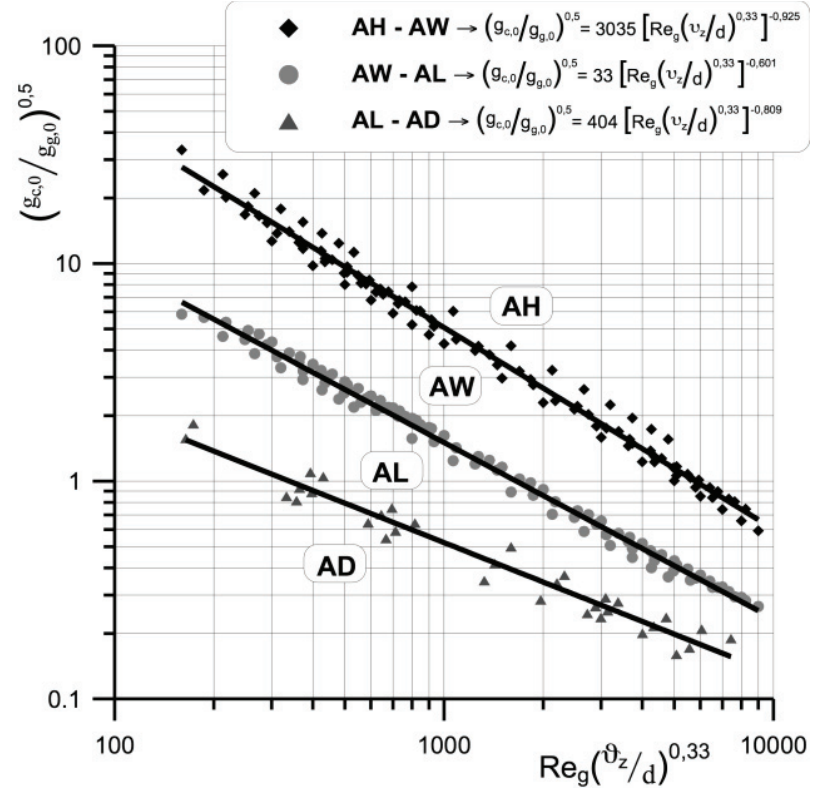

Fig. 8. Universal map of two-phase gas-very viscous liquid downward flow in a pipe.
From the point of view of hydrodynamics of downward flowing liquid, with regard to both theoretical research and conducted analysis, it was interesting to record parameters such as forming liquid films, state of the waves on its surface and value of the interfacial surface. In accordance with the adopted methodology, the measurement of liquid film thickness was undertaken using optoelectronic methods, both with regard to linear (point) and planar system of measurement.

Research involved determination of the effect of flow parameters on the variability of measured values with a particular emphasis on the effect of the variable liquid viscosity. The thickness identified during studies - for the reasons of the applied measurement systems - was determined for local states and averaged for specific flow conditions. This, in turn, enabled one to assess the nature of the downward flowing film. Independently, trap method was applied, whose results provided the results of mean liquid thickness over the entire length of the channel. In each of these cases the averaged local states were integrated means referred to time and length of the measurement channel.

From the analysis of the results one can conclude that the hydrodynamics of annular gas-liquid flow is mostly affected by liquid viscosity and gas stream. An increase in the velocity of the liquid always results in formation of film with greater thickness while an increase in the velocity of the gas phase for a constant liquid flow rate per unit periphery usually leads to a decrease in the oil film thickness. This tendency was observed with regard to all examined pipe diameters over the total range of changing viscosities. One can note, however, that this tendency is particularly discernible for turbulent flow of the gas phase. However, over the range of small velocities of the gas phase, the thickness of liquid film is close to the value specific for its gravitational flow. This is illustrated by the results of research presented in Figs. 9-10, whose additional interpretation (in 3D) is presented in Figs. 11 to 12.

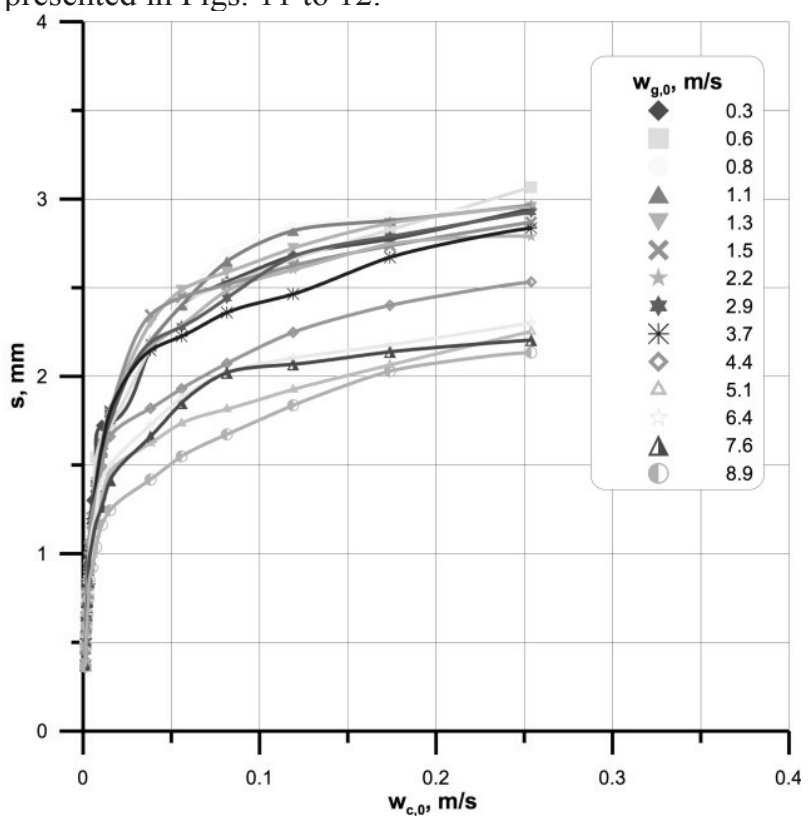

Fig. 9. Distribution of experimental points thickness of liquid film $\eta_{\mathrm{c}}=90 \mathrm{mPa} \cdot \mathrm{s}$. 
These results describe the characteristics of film thickness variability observed during annular flow of oil for various flow conditions.

From the distribution of experimental points (Figs. 9-10) it stems that an increase in superficial velocity of gas does not always promote a decrease in oil film thickness and that the viscosity of the liquid and its superficial velocity affects it.

An increase of the viscosity of the liquid forms a reason for formation of its thicker film, which tends to increase faster following an increase in the liquid stream.

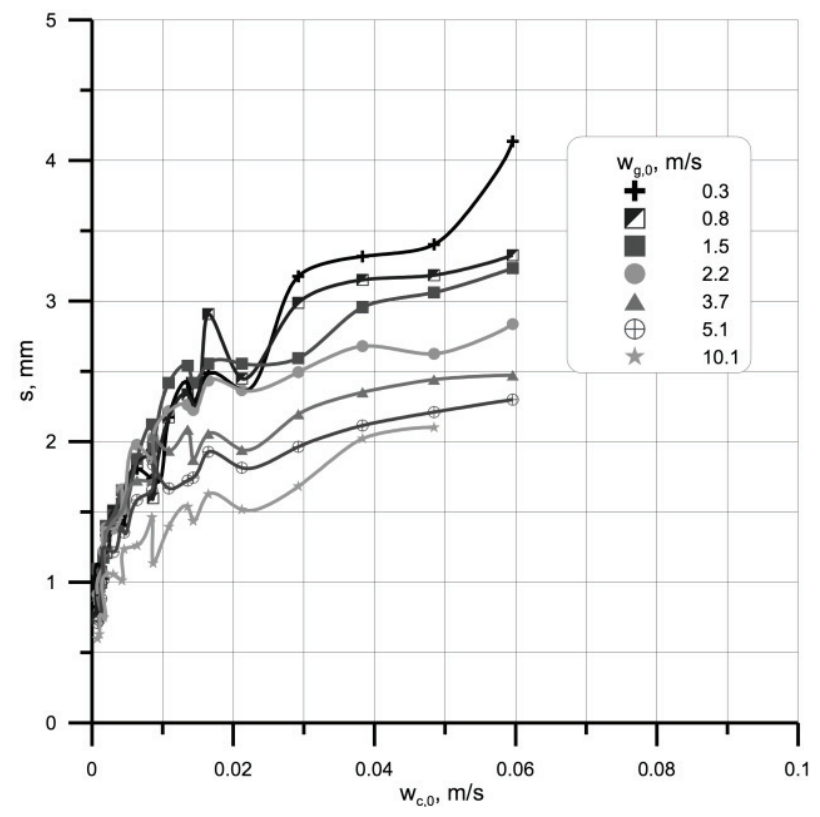

Fig. 10. Distribution of experimental points characterizing thickness of liquid film $\eta_{\mathrm{c}}=2200 \mathrm{mPa} \cdot \mathrm{s}$.

A direct increase of the liquid's superficial velocity (for remaining parameters remaining constant) promotes greater wavy structure in the interfacial surface (Figs. 11-12), which consequently leads to formation of hydraulic flow with increasingly curvy interfacial surface.

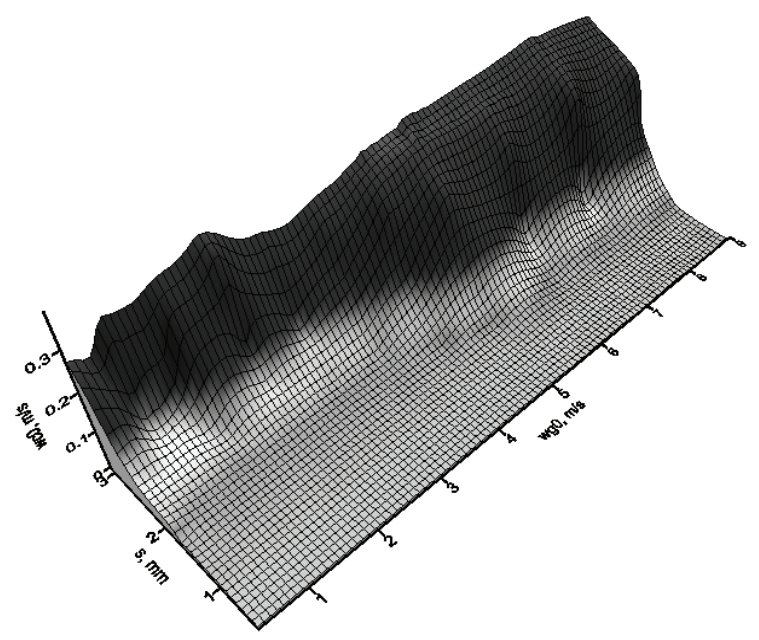

Fig. 11. Surface representation of variability of liquid film thickness $\eta_{\mathrm{c}}=90 \mathrm{mPa} \cdot \mathrm{s}$ (respective to Fig. 9).
An additional illustration of these types of phenomena is found in Figs. 13-14, which contain a set of exemplary profiles of changes in the thickness of the oil film with respect to Reynolds number for gas and liquid.

The distribution of the lines of these profiles indicates that for the highest gas streams (Fig. 14), regardless of liquid flow rate per unit periphery we will always have to do with hydraulic forms of viscous liquid flow, while in the remaining cases the transfer to such a form is considerably relative to the liquid stream.

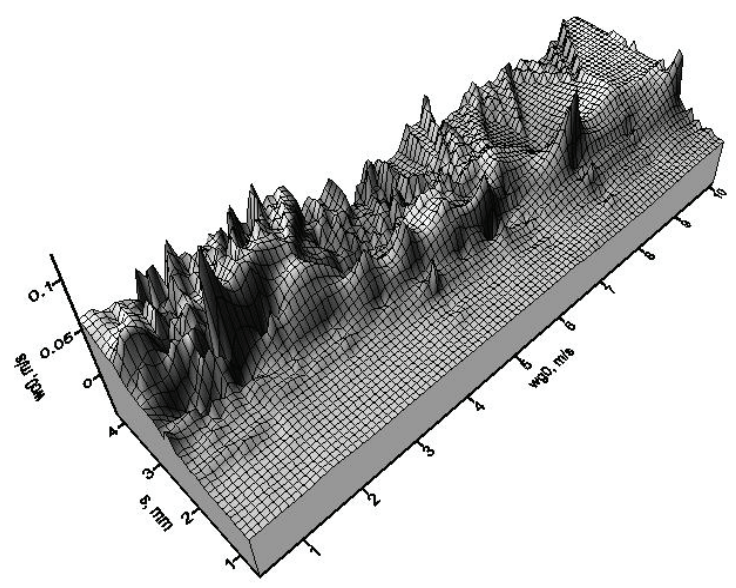

Fig. 12. Surface representation of variability of liquid film thickness $\eta_{\mathrm{c}}=1070 \mathrm{mPa} \cdot \mathrm{s}$ (respective to Fig. 10).

The relatively low values of it lead to the formation of wave structures in the flow (Figs. 13-14), which transform into hydraulic flow along with an increase of the gas stream.

It was concurrently concluded that the relative wave velocities (in relation to the mean velocity of the liquid film) tends to considerably drop following an increase of equivalent Reynolds number.
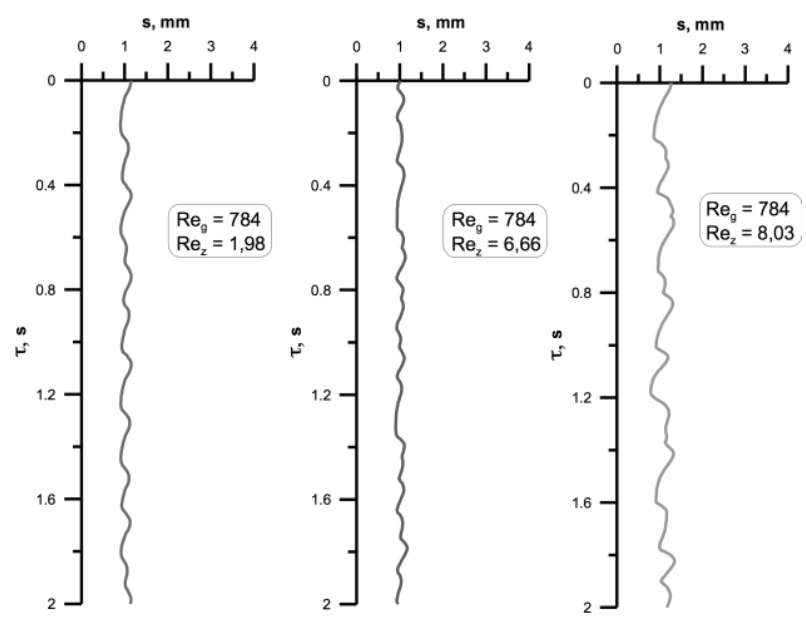

Fig. 13. Representation of variable liquid film thickness along the flow route: $\left(D=22 \mathrm{~mm}, \mathrm{Re}_{\mathrm{g}}=785, \eta_{\mathrm{c}}=1070 \mathrm{mPa} \cdot \mathrm{s}\right)$.

The interpretation of the research results indicates a considerable tendency of variation of the relation $\mathrm{F}_{2 \mathrm{~F}} / \mathrm{F}_{\mathrm{p}}$ with respect to void fractions resulting from the criteria 
involving criteria based Reynolds number for liquid and gas, as presented in Fig. 15. From the distribution of the experimental points it stems that for a constant value of $\mathrm{Re}_{\mathrm{g}}$ along with an increase of the value of equivalent Reynolds number we have to do with considerable decrease in the value of the actual contact surface on the boundary of the phases. This results from a concurrent rise of liquid film thickness (for increasingly higher $R_{z}$ ) on one hand, and on the other, from the changes in the characteristics of waves of downward liquid film flow for higher liquid volumes, which is particularly discernible for very wavy and hydraulic annular flow structures.
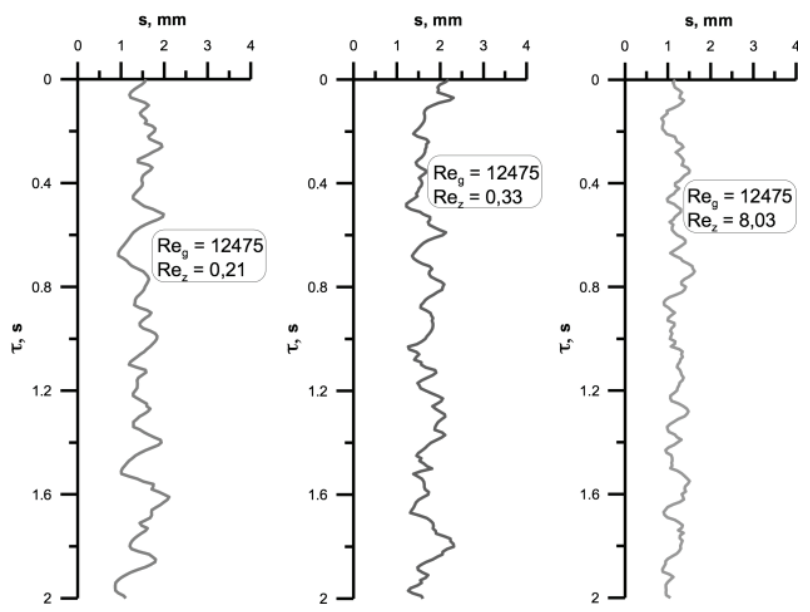

Fig.14. Representation of variable liquid film thickness along the flow route: $\left(D=22 \mathrm{~mm}, \mathrm{Re}_{\mathrm{g}}=12474, \eta_{\mathrm{c}}=1070 \mathrm{mPa} \cdot \mathrm{s}\right)$.

Additionally, as presented in Fig. 15 in these flow conditions an important role can be attributed to the characteristics of the gas phase.

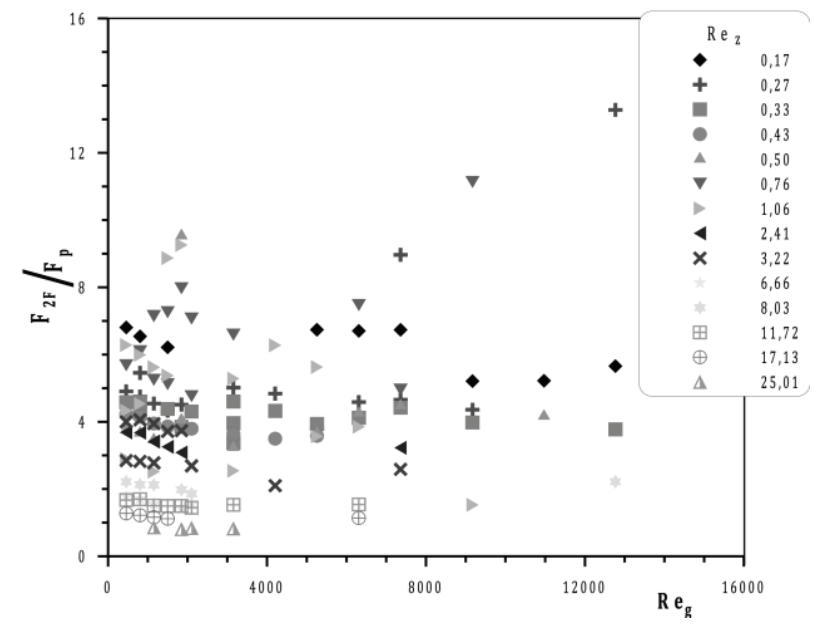

Fig. 15. Relative value of interfacial surface $\left(F_{2 F} / F_{p}\right)$ for various process conditions.

Whereas for the area of laminar flow we can observe relatively proportional increase of the relation $F_{2 F} / F_{r}$ for decreasing equivalent Reynolds number $\left(\operatorname{Re}_{\mathrm{z}}\right)$, for the case of the turbulent flow of this phase (e.g. with regard to strongly wavy films and hydraulic flow) such an increase is not very unequivocal.
A closer investigation of the results has indicated that it is possible to determine the characteristics of these changes relative to any interpreted void fraction both in accordance with (1) and (2). Hence, just as referred to in the case of film thickness, a proposition is made to apply two alternative relations in order to determine the interfacial surface in two-phase gas-very viscous liquid flow. These are:

a) for adiabatic conditions

$$
\frac{F_{2 F}}{F_{p}}=47.3\left(\frac{\varepsilon}{1-\varepsilon}\right)^{0.636} R e_{g}^{-0.683} R e_{Z}^{-0.109}
$$

b) with respect to mass void fraction - one that is more beneficial in terms of conditions of heat exchange

$\frac{F_{2 F}}{F_{p}}=2.85 \cdot 10^{3}\left(\frac{x}{1-x}\right)^{0.627} R e_{g}^{-0.669} R e_{c}^{-0.111}$

where:

$\operatorname{Re}_{\mathrm{z}}=4 \Gamma / \eta_{\mathrm{c}}, \Gamma=\dot{\mathrm{m}}_{\mathrm{c}} / \pi \mathrm{d}, \mathrm{Re}_{\mathrm{g}}=\mathrm{g}_{\mathrm{T}} \mathrm{xD} / \eta_{\mathrm{g}}$,

$\operatorname{Re}_{\mathrm{c}}=\mathrm{g}_{\mathrm{T}}(1-\mathrm{x}) \mathrm{D} / \eta_{\mathrm{c}}, \varepsilon=\mathrm{V}_{\mathrm{g}} /\left(\mathrm{V}_{\mathrm{g}}+\mathrm{V}_{\mathrm{c}}\right), \mathrm{x}=\mathrm{g}_{\mathrm{g}} /\left(\mathrm{g}_{\mathrm{g}}+\mathrm{g}_{\mathrm{c}}\right)$

A comparison between the results of measured interfacial surface and ones calculated in accordance with (1) and (2) indicates that over $85 \%$ of points are found to be within the boundaries of $\pm 15 \%$ relative error (for the correlation coefficient of over 0.95 in both cases). For relation (2) it is illustrated in Fig. 16. Therefore, one can assume that these relations serve for a satisfactory description of interfacial surface, which is encountered for co-current downward flow of gas-very viscous liquid in vertical pipes.

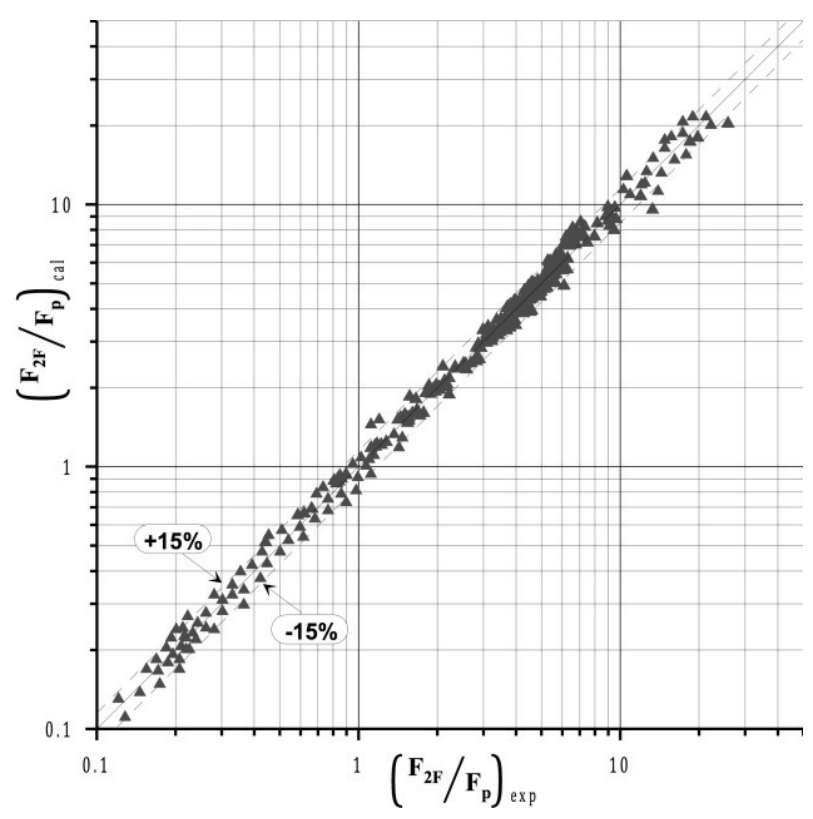

Fig. 16. Distribution of experimental points - in accordance with (2).

\section{Summary}

On the basis of analysis of the identified annular downward flow structures of viscous liquid flow determined during experiment a conclusion has been 
made that the forms of annular structure can differ considerably depending on liquid viscosity and mutual relations of velocities of two phases - from smooth liquid film to severely dispersed hydraulic form. Finally, for this flow regime a classification of flow structures found in Fig. 4 is proposed. In general, this classification reflects the characteristics of annular gas-very viscous liquid two-phase flow and involves identification of structures in downward flow of oil-air mixture in a vertical pipe with a diameter of $22 \mathrm{~mm}$ within the range of viscosity coefficient of (90-3500) $\mathrm{mPa} \cdot \mathrm{s}$.

Accounting for the similarity of structures formed, for the phenomenological assessment three boundaries at which different flow patterns emerging for annular gasvery viscous liquid two-phase flow are distinguished, which has enabled these authors to present a universal flow structure map found in Fig. 8.

On the basis of assessing the effect of specific process parameters on forming of qualitatively different forms of annular flow, a conclusion is made that liquid viscosity forms the dominant parameter affecting particular structure formation. In consideration of the fact that this map has been developed for a relatively small range of variations in process parameters, one can conclude that it is can fully applied with regard to calculations of tubular thin film apparatuses, in which the annular gas-very viscous liquid flow.

It was additionally indicated that an increase in the velocity of gas flow facilitates the origin of increasingly greater changes on the surface of liquid film. Except for films, which are smooth or slightly wavy, these changes are irregular and are demonstrated by various development of interfacial surface. The waves and turbulence found on the separation surface have amplitudes and wavelengths that are time variable and spatially varied. This has made the quantitative description of hydrodynamics of such flow even more complicated.

From the analysis of experimental data it stems that within the entire range of process parameters all of them have a considerable effect on the formation of liquid film. An increase in the viscosity of the liquid always promotes an increase of the mean thickness of liquid layer, while increase of the gas phase steam for a constant liquid flow rate per unit periphery generally results in a decrease of the film thickness. This, however, occurs only in the area of the flow with strongly developed interfacial surface, just as it takes place in hydraulic flow. These conditions are often accompanied by the phenomenon of wave interference, which promotes their accumulation or results in formation of additional capillary waves, which in turn, brings their greater local thickness (in comparison to mean values). This idiosyncrasy is especially characteristic for a liquid with a high viscosity.

In the relations forming the model of hydrodynamics of two-phase gas-very viscous liquid flow the wide range of variability of liquid viscosity coefficient $(90 \div 3500)$ $\mathrm{mPa} \cdot \mathrm{s}$ is covered, which has not been fully considered in literature of this subject yet. This work involves a precise verification of experimental data, which can promote qualitative and quantitative assessment of flow phenomena that accompany such flow conditions. The scope of this research also enables one to recommend the developed model for engineering and applied design work in assessing the operation of thin film equipment with hydraulically generated liquid film.

\section{Nomenclature}

$F$ - surface, $\mathrm{m}^{2}$

$R e$ - Reynolds number,

$V$ - volume flow rate, $\mathrm{m}^{3} / \mathrm{s}$

$d$-diameter, $\mathrm{m}$

$g$-mass flux, $\mathrm{kg} /\left(\mathrm{m}^{2} \mathrm{~s}\right)$

$\dot{m}$ - mass flow, $\mathrm{kg} / \mathrm{s}$

$s$ - liquid film thickness, $\mathrm{m}$

$\Gamma$-wetting rate, $\mathrm{kg} /(\mathrm{ms})$

$\eta$-viscosity, $\mathrm{Pa} \cdot \mathrm{s}$

$\rho$ - density, $\mathrm{kg} / \mathrm{m}^{3}$

$\vartheta_{z}$ - equivalent linear dimension, $\mathrm{m}$

\section{Subscripts and superscripts}

0 - superficial (calculated for full cross-section area),

$\mathrm{c}-$ liquid,

cal - calculated,

exp - experimental,

$\mathrm{g}$ - gas,

$\mathrm{p}$ - pipe internal surface area,

$\mathrm{Z}$ - equivalent values for free falling liquids

2F- two-phase flow.

\section{References}

1. N. Andritsos, T. J. Hanratty, Int. J. of Mult. Flow, 13, 5 (1987)

2. K. Czernek, Chem. Eng. and Equ., 6 (2009)

3. K. Czernek, S. Witczak, Chem. and Proc. Eng., 34 (2013)

4. K. Czernek, Studies and Monographs 347, Opole, (2013)

5. K. Czernek, G. Filipczak, S. Witczak, Przem. Chem., 87/2, (2008)

6. M.J. Da Silva, S. Thiele, L. Abdulkareem, B.J. Azzopardi, U. Hampel, Flow Meas. and Instr., Volume 21, 3 (2010)

7. G. Geraci, B.J. Azzopardi, H.R.E. van Maanen, Chem. Eng. Sc., 62, 11 (2007)

8. V. Hernandez Perez, B.J. Azzopardi, R. Kaji, M.J. da Silva, M. Beyer, U. Hampel, Int. J. of Mult. Flow, 36, 11-12 (2010)

9. E.W. Jassim, T.A. Newell, J.C. Chato, Exp. Therm. and Fluid Sc., 32, 5 (2008)

10. J. Oriol, J.P. Leclerc, C. Jallut, P. Tochon, P. Clement, Chem. Eng. Sc., 63, 1 (2008)

11. L. Szalinski, L.A. Abdulkareem, M.J. Da Silva, S. Thiele, M. Beyer, D. Lucas, V. Hernandez Perez, U. Hampel, B.J. Azzopardi, Chem. Eng. Sc., 65, 12 (2010)

12. L. Troniewski, S. Witczak, K. Czernek, Chem. and Proc. Eng., 27 (2006) 
13. L. Troniewski, W. Spisak, Int. J. of Mult. Flow, 13, 2 (1987)

14. S. Witczak, K. Czernek, Turbulence, 11 (2005)

15. J.Y Xu, Y.X Wu, Z.H Shi, L.Y Lao, D.H Li, Int. J. of Mult. Flow, 33, 9 (2007) 